\title{
Linear multi-hop amplify-and-forward (AF) cooperative relay channels
}

\begin{abstract}
Cooperative relaying of channels between base station and user equipment emerged as a great technique to enhance the reliability and throughput of cellular networks. The relay nodes normally exploit amplify-and-forward (AF) scheme to relay the connection. Although there have been many previous works on cooperative issues, none of them have considered the fundamental trade-off between the communication reliability and transmission rate. Instead of considering the performance evaluated separately on the achievable rate or the error probability, the RCEE provide insight into fundamental trade-off between the transmission rate and the communication reliability in cooperative relay channels. It has been shown that multi-hop relay nodes are necessary to compensate the decreasing SNR over the distance. As a trade-off, the communication reliability in terms of RCEE is decreasing over the increase of multi-hop relay nodes with low SNR for a given data rate. Therefore, the optimization for the number of hops is preferable in the cooperative relay channels. Cooperative relaying of channels between base station and user equipment emerged as a great technique to enhance the reliability and throughput of cellular networks. The relay nodes normally exploit amplifyand-forward (AF) scheme to relay the connection. Although there have been many previous works on cooperative issues, none of them have considered the fundamental trade-off between the communication reliability and transmission rate. Instead of considering the performance evaluated separately on the achievable rate or the error probability, the RCEE provide insight into fundamental trade-off between the transmission rate and the communication reliability in cooperative relay channels. It has been shown that multi-hop relay nodes are necessary to compensate the decreasing SNR over the distance. As a tradeoff, the communication reliability in terms of RCEE is decreasing over the increase of multihop relay nodes with low SNR for a given data rate. Therefore, the optimization for the number of hops is preferable in the cooperative relay channels.
\end{abstract}

Keyword: Amplify-and-forward; Cooperative; Linear multi-hop relay channels; Random coding error exponent; Signal noise ratio 